Editorial

\title{
ICD-I0 and DSM-5 differences may explain lack of psychiatric progress
}

Volume 7 Issue 2 - 2017

\section{Editorial}

Modern psychiatry is a harbor of "possibly interconnected" twin realities that may help reflect challenges faced by millions with psychiatric disorders.

The first observation is that, the predominant diagnostic instrument of modern psychiatry the Diagnostic Statistical Manual of Mental Disorders fifth edition (DSM 5) has subtle and crucial differences from the International Classification of Diseases 10th edition (ICD 10):

i. ICD is built upon biological sciences, anatomy and pathophysiology.

ii. DSM Is not.

iii. ICD includes data from biochemical assays and Imaging.

iv. DSM does not.

v. ICD differentiates complication from illness.

vi. DSM doesn't.

vii. ICD is an accurate diagnostic map.

viii. DSM is not.

The second, is the lack of any hard evidence that scientific discoveries have improved general mental health or lessened suffering. For, several crucial benchmarks of psychiatric progress i.e. deaths by suicide and overdoses by illicit drugs have been rising at a time when crime rates and homicides have been on a steady decline.

DSM has influence: it dictates treatment, research and health policy. Reason says DSM induced inappropriate treatment, wasteful research and ill-advised public health policy might have contributed to the current reality.

DSM 5 flaws have remained off scientific radar partly because they are what physics call eigenvalues: real phenomenon -such as electricity or magnetic fields - that are not easily observable and often invisible.

DSM is simple and it makes it possible for anyone with a high school education to diagnose highly complex medical conditions. Simplicity is not a bad thing for a diagnostic instrument. However

\author{
Alen J Salerian \\ Neuroscience Institute, USA
}

Correspondence: Alen J Salerian, Neuroscience Institute, 8409 Carlynn Dr. Bethesda, MD 208I7, USA, Tel 30I-204-9004, Email alensalerian@gmail.com

Received: January 14, 2017 | Published: January 23, 2017

a simple, popular and also inaccurate instrument could pose exponentially heightened dangers. DSM 5 lets medical disorders become diagnostic codes of signs and symptoms without supporting evidence or biologically meaningful connections.

The end result? Major gains against deaths by cancer, cardiovascular diseases but not from alcoholism or depression.

There is an urgent need to study possible adverse effects of DSM 5. If necessary, we must develop a scientifically valid and safe diagnostic instrument in psychiatry.

Will psychiatry have the will not to simply upgrade DSM 5 by patchwork? Why not to design a diagnostic tool that could accommodate the dynamic complexities of brain function and psychiatric disorders?

\section{Acknowledgments}

None.

\section{Conflicts of interest}

Author declares there are no conflicts of interest.

\section{Funding}

None. 